\title{
Global Stability Analysis of Switched Nonlinear Observers
}

\author{
Imen Manaa Nabil Barhoumi Faouzi Msahli \\ The National Engineering School of Monastir, Department of Electrical Engineering, Study of Industrial Systems and Renewable Energy, \\ Monastir 5019, Tunisia
}

\begin{abstract}
This paper considers the problem of simultaneous estimation of the system states and the strategy of commutation for a larger class of nonlinear switched systems. First, a hybrid high gain observer is considered to get the exact estimation of the continuous states where the strategy of switching is previously known. Then, an extension to a larger class of nonlinear hybrid systems with arbitrary switching is made. Stability analysis is widely discussed for the two cases to provide a finite-time convergence of the estimation errors. The effectiveness of the proposed hybrid high gain observer has been proved by applying it to a quadruple tank process.
\end{abstract}

Keywords: Nonlinear hybrid system, switching strategies, Lyapunov function, stability analysis.

\section{Introduction}

Nowadays, several physical processes, such as chemical, electrical and mechanical engineering systems, cannot be modeled considering exclusively continuous or discrete dynamics. Many efforts have been made to describe the interaction between continuous and discrete behaviors which involve the hybrid dynamics of the systems ${ }^{[1-3]}$. Switched systems are a particular class of systems that constitute a set of continuous subsystems and a switching signal orchestrating the commutation between them ${ }^{[4-6]}$.

During the last decades, the state estimation problem for switched hybrid systems has attracted a lot of attention. In the linear case, several hybrid state estimation methods have been proposed in the literature depending on the knowledge of the active mode. Thus, an approach of Luenberger observers has been presented when the mode location is known. The related stabilizing gains calculation as a linear matrix inequality approach by using multiple Lyapunov functions are considered ${ }^{[7,8]}$. With the same conditions, Juloski et al. ${ }^{[9]}$ proposed two types of linear observers, based on the prediction errors. An estimation structure for the class of piecewise system has been proposed in [10]. A study of an observer-based stabilization of switching linear systems where the dynamics of each mode are known a priori is addressed in $[11,12]$. In particular case, when the active mode is unknown, the design of continuous-discrete linear observer for hybrid systems has been presented in $[13,14]$.

In the nonlinear case, the solution to the problem of how to combine established techniques for discrete and continuous nonlinear systems to develop different methods of observation has became a necessity. Hence, some recent results of research are obtained for the synthesis of observers for hybrid systems. A step by step sliding mode observer has

\footnotetext{
Regular Paper

Manuscript received January 1, 2014; accepted April 2, 2014

Recommended by Associate Editor Wing Cheong Daniel Ho

(C) Institute of Automation, Chinese Academy of Science and Springer-Verlag Berlin Heidelberg 2015
}

been considered in [15] to estimate continuous and discrete states without jump and excluding the Zeno phenomena. Saadaoui et al. ${ }^{[16,17]}$ used a nonlinear observer for the class of switched Lagrangian system that can be described in the observer canonical form with fixed switching strategy.

The purpose of the present work is to design an observer for a large class of uniformly observable hybrid systems by extending the results of the frameworks based on continuous $^{[18-20]}$ and discrete systems ${ }^{[21]}$. According to our first case, we will design an algorithm of full order high gain observer for simple continuous state estimation supposing that the switching strategy, also called discrete location, is previously known. In another case, we will propose a discrete-continuous observer for the presented class of systems. Therefore, the discrete mode design is identified according to the estimated switching strategy law and the continuous state is then estimated. The main contribution of this paper concerns the global stability analysis for the two cases: studied and proved by means of multiple Lyapunov functions.

In such class of systems, the choice of initial conditions of the states for every subsystem is an obligation to avoid the chattering phenomena during the transient period.

This paper is organized as follows. Section 2 is dedicated to introduce the problem statement and the class of the switched multiple-input multiple-output (MIMO) nonlinear hybrid systems. In Section 3, the hybrid observer with known switching strategy is developed and stability analysis is carried out. Then, an extension to a discretecontinuous observer design is made and a global stability analysis is detailed. In Section 4, an illustrative example is treated to show the effectiveness of design methodology. Simulation results are carried out to evaluate the robustness of the proposed observer. Finally, some conclusions are presented in the last section. 


\section{Problem statement}

In this study, our aim is to address the problem of MIMO uniformly observable hybrid systems. The dynamical behavior of such class of systems can be described by the state representation as

$$
\sum_{\sigma}\left\{\begin{array}{l}
\dot{x}(t)=A x(t)+\varphi_{\sigma}(u(t), x(t)) \\
y_{\sigma}(t)=C x(t)=x^{1}
\end{array}\right.
$$

A set of admissible switching strategy of the system is defined by a piecewise constant function: $\sigma(t) \in \prod$ $=\{1,2, \cdots, M\}$. It is represented by $\sigma=\left\{\tau_{i}, \sigma\left(\tau_{i}\right)\right\}_{i=1}^{M}$ and indicates the instant of the switching system from subsystem $\sigma\left(\tau_{i-1}\right)$ to subsystem $\sigma\left(\tau_{i}\right)$. During the time interval $\left[\tau_{i}, \tau_{i+1}\right]$, the subsystem $\sigma\left(\tau_{i}\right)$ is active. We assume that each subsystem is uniformly observable.

The states are $x(t) \in \mathbf{R}^{n}, x^{k} \in \mathbf{R}^{n_{k}}, \sum_{k=1}^{p} n_{k}=n, k=$ $1, \cdots, q$, the input $u \in U \subset \mathbf{R}^{m}$ with $m \geq p$. A, C, $x$ and $\varphi_{\sigma}$ are such that

$$
A=\left[\begin{array}{ccccc}
0 & I_{p} & 0 & \cdots & 0 \\
0 & \cdots & I_{p} & \cdots & \cdots \\
\cdots & \cdots & \cdots & \cdots & 0 \\
\cdots & \cdots & \cdots & \cdots & I_{p} \\
0 & \cdots & \cdots & 0 & 0
\end{array}\right], C=\left(\begin{array}{ll}
I_{p} & 0_{p} \cdots 0_{p}
\end{array}\right)
$$

$$
\begin{gathered}
x=\left(\begin{array}{c}
x^{1} \\
\vdots \\
x^{q}
\end{array}\right), x^{k}=\left(\begin{array}{c}
x_{1, \sigma}^{k} \\
x_{2, \sigma}^{k} \\
\vdots \\
x_{p, \sigma}^{k}
\end{array}\right) \\
\varphi_{\sigma}(x)=\left(\begin{array}{c}
\varphi_{\sigma}^{1}\left(u, x^{1}\right) \\
\varphi_{\sigma}^{2}\left(u, x^{1}, x^{2}\right) \\
\vdots \\
\varphi_{\sigma}{ }^{q-1}\left(u, x^{1}, \cdots, x^{q-1}\right) \\
\varphi_{\sigma}^{q}(x)
\end{array}\right)
\end{gathered}
$$

The following assumptions will be made.

Assumption 1. $\varphi_{\sigma}(u, x)$ is a globally Lipschitz nonlinear function with respect to $x$ uniformly in $u_{\sigma}$.

Assumption 2. There exists a positive real number $\tau_{\min }$ defined by

$$
\tau_{i+1}-\tau_{i} \geq \tau_{\min }>0, \quad i \in \Pi=\{1,2, \cdots, M\}
$$

where $\tau_{i+1}-\tau_{i}$ defines the dwell time between two switches which is supposed to be sufficient to allow the exponential convergence of the observer to the real system before a new switch. In fact, the Zeno phenomena in the interval $\left[t_{0}, t_{f}\right]$ is excluded.

Assumption 3. There exists a positive constant $\rho$ such that $\left|\varphi_{\hat{\sigma}}\left(x_{\sigma}\right)-\varphi_{\sigma}\left(x_{\sigma}\right)\right| \leq \rho$ is bounded for all $(\sigma, \hat{\sigma}) \in \Pi$, $x \in \mathbf{R}^{n}$.

\section{Observer synthesis}

\subsection{Observer design with fixed switching strategy}

The continuous observer for the nonlinear switched hybrid systems class (1) is given by

$$
\dot{\hat{x}}(t)=A \hat{x}(t)+\varphi_{\sigma}(\hat{x}(t), u)-\theta_{\sigma} \Delta_{\theta_{\sigma}}^{-1} S^{-1} C^{\mathrm{T}}(C \hat{x}(t)-y(t))
$$

where $A$ and $C$ are given by (2). $\theta_{\sigma} \geq 0$ is a real number. Let $\Delta_{\theta_{\sigma}}$ be the block diagonal matrix defined by

$$
\Delta_{\theta_{\sigma}}=\operatorname{diag}\left[\begin{array}{llll}
I_{p} & \frac{I_{p}}{\theta_{\sigma}} & \cdots & \frac{I_{p}}{\theta_{\sigma}^{n}}
\end{array}\right] .
$$

Let $S$ be the unique positive definite solution of the algebraic Lyapunov equation as

$$
S+A^{\mathrm{T}} S+S A-C^{\mathrm{T}} C=0 .
$$

The solution of (7) is

$$
S^{-1} C^{\mathrm{T}}=\left(C_{q}^{1} I_{p}, \cdots, C_{q}^{q} I_{p}\right)
$$

with $C_{q}^{i}=\frac{q !}{i !(q-1) !}$ for $i=1, \cdots, q$.

Theorem 1. If the nonlinear switched hybrid system defined by (1) satisfies the Assumptions 1 and 2, then the observer described by (5) leads to a global exponential convergence of the estimation error for fixed $\sigma$.

Proof. In this case, we define the exponential convergence problem of the whole switched hybrid system supposing that the switching strategies $\sigma$ to be known. We set $e_{\sigma}(t)=\hat{x}_{\sigma}(t)-x_{\sigma}(t)$ to be the vector of the error between the real state vector and its estimation. We have

$$
\begin{aligned}
\dot{e}_{\sigma}(t)= & A e_{\sigma}(t)-\theta_{\sigma} \Delta_{\theta_{\sigma}}^{-1} S^{-1} C^{\mathrm{T}}(C \hat{x}(t)-y(t))+ \\
& \varphi_{\sigma}(\hat{x}(t), u)-\varphi_{\sigma}(x(t), u) .
\end{aligned}
$$

We can easily verify the following identities: $\Delta_{\theta_{\sigma}} A \Delta_{\theta_{\sigma}}^{-1}=$ $\theta_{\sigma} A$ and $C \Delta_{\theta_{\sigma}}=C$.

We consider the following change of variable $\bar{e}_{\sigma}=\Delta_{\theta_{\sigma}} e_{\sigma}$. $\dot{\bar{e}}_{\sigma}=\theta_{\sigma} A \bar{e}_{\sigma}-\theta_{\sigma} S^{-1} C^{\mathrm{T}} C \bar{e}_{\sigma}+\Delta_{\theta_{\sigma}}\left(\varphi_{\sigma}(\hat{x}, u)-\varphi_{\sigma}(x, u)\right)$.

$V_{\sigma}$ is the Lyapunov function candidate with $V_{\sigma}=\bar{e}_{\sigma}^{\mathrm{T}} S \bar{e}_{\sigma}$.

$$
\begin{aligned}
\dot{V}_{\sigma}= & 2 \bar{e}_{\sigma}^{\mathrm{T}} S \dot{\bar{e}}_{\sigma}= \\
& 2 \theta_{\sigma} \bar{e}_{\sigma}^{\mathrm{T}} S A \bar{e}_{\sigma}-2 \theta_{\sigma} \bar{e}_{\sigma}^{\mathrm{T}} C^{\mathrm{T}} C \bar{e}_{\sigma}+ \\
& 2{\overline{e_{\sigma}}}^{\mathrm{T}} S \Delta_{\theta_{\sigma}}\left(\varphi_{\sigma}(\hat{x}, u)-\varphi_{\sigma}(x, u)\right) .
\end{aligned}
$$

Using (7), we can show that $S$ is symmetric and positive definite where $A$ and $C$ are respectively given by (2). We have $2 S A=C^{\mathrm{T}} C-S$.

$$
\begin{aligned}
\dot{V}_{\sigma}= & -\theta_{\sigma} V_{\sigma}+\theta_{\sigma} \bar{e}_{\sigma}^{\mathrm{T}} C^{\mathrm{T}} C \bar{e}_{\sigma}-2 \theta_{\sigma} \bar{e}_{\sigma}^{\mathrm{T}} C^{\mathrm{T}} C \bar{e}_{\sigma}+ \\
& 2 \bar{e}_{\sigma}^{\mathrm{T}} S \Delta_{\theta_{\sigma}}\left(\varphi_{\sigma}(\hat{x}, u)-\varphi_{\sigma}(x, u)\right) \\
\dot{V}_{\sigma}= & -\theta_{\sigma} V_{\sigma}+2 \theta_{\sigma}\left(\frac{1}{2} \bar{e}_{\sigma}^{\mathrm{T}} C^{\mathrm{T}} C \bar{e}-\bar{e}_{\sigma}^{\mathrm{T}} C^{\mathrm{T}} C \bar{e}_{\sigma}\right)+ \\
& 2 \bar{e}_{\sigma}^{\mathrm{T}} S \Delta_{\theta_{\sigma}}\left(\varphi_{\sigma}(\hat{x}, u)-\varphi_{\sigma}(x, u)\right) \leq \\
& -\theta_{\sigma} V_{\sigma}+2 \bar{e}_{\sigma}^{\mathrm{T}} S \Delta_{\theta_{\sigma}}\left(\varphi_{\sigma}(\hat{x}, u)-\varphi_{\sigma}(x, u)\right) .
\end{aligned}
$$


Let $\delta=\max _{\sigma \in \Pi}\left\{\delta_{\sigma}\right\}$ and $\theta_{\sigma} \geq 1$. Based on the Assumption 1, we can deduce

$$
\left\|\Delta_{\theta_{\sigma}}\left(\varphi_{\sigma}(\hat{x}, u)-\varphi_{\sigma}(x, u)\right)\right\| \leq \delta_{\sigma}\left\|\bar{e}_{\sigma}\right\| \leq \delta\left\|\bar{e}_{\sigma}\right\|
$$

where $\lambda_{\max }(S)$ and $\lambda_{\min }(S)$ are the maximal and minimal eigenvalues of $S$, respectively. We obtain

$$
\begin{aligned}
\dot{V}_{\sigma}\left(\bar{e}_{\sigma}\right) \leq & -\theta_{\sigma} V_{\sigma}+2 \lambda_{\max }(S)\left\|\bar{e}_{\sigma}\right\| \delta\left\|\bar{e}_{\sigma}\right\| \leq \\
& -\theta_{\sigma} V_{\sigma}+2 \frac{\delta \lambda_{\max }(S)}{\lambda_{\min }(S)} V_{\sigma}
\end{aligned}
$$

Supposing that $\alpha=2 \frac{\delta \lambda_{\max }(S)}{\lambda_{\min }(S)}$, we find

$$
\dot{V}_{\sigma} \leq-\left(\theta_{\sigma}-\alpha\right) V_{\sigma} \leq-\mu_{\sigma} V_{\sigma} \leq-\mu V_{\sigma}
$$

where $\mu=\inf _{\sigma \in \Pi}\left(\theta_{\sigma}-\alpha\right)>0$ and $\alpha<\min \left(\theta_{\sigma}\right)$.

Therefore,

$$
\begin{gathered}
V_{\sigma}\left(\bar{e}_{\sigma}\right) \leq \mathrm{e}^{-\left(\theta_{\sigma}-\alpha\right)\left(t-t_{0}\right)} V_{\sigma}\left(\bar{e}_{\sigma}(0)\right) \leq \\
\mathrm{e}^{-\mu\left(t-t_{0}\right)} V_{\sigma}\left(\bar{e}_{\sigma}(0)\right) .
\end{gathered}
$$

For $t \in\left[\tau_{i-1}, \tau_{i}\right]$ and satisfying the Assumption 2, we get

$$
V_{\sigma\left(\tau_{i}^{-}\right)} \leq \mathrm{e}^{-\mu\left(\tau_{i}-\tau_{i-1}\right)} V_{\sigma\left(\tau_{i-1}^{+}\right)} .
$$

For $t \in\left[\tau_{i}, \tau_{i+1}\right]$,

$$
V_{\sigma\left(\tau_{i+1}^{-}\right)} \leq \mathrm{e}^{-\mu\left(\tau_{i+1}-\tau_{i}\right)} V_{\sigma\left(\tau_{i}^{+}\right)} .
$$

We have to ensure the global stability of the error dynamics during the switching from one subsystem to another. It is assumed, then, that $e_{\sigma}\left(\tau_{i}^{+}\right)=\Gamma e_{\sigma}\left(\tau_{i}^{-}\right) . \Gamma$ is a positive definite $n$-dimensional matrix. In a particular case, $\Gamma=I$ which means that we have no jumps. Hence, we get

$$
\begin{aligned}
V_{\sigma\left(\tau_{i}^{+}\right)}= & \bar{e}_{\sigma}^{\mathrm{T}} \bar{S} \bar{e}_{\sigma}=\left(\Delta_{\theta_{\sigma}} e_{\sigma}\right)^{\mathrm{T}} \bar{S}\left(\Delta_{\theta_{\sigma}} e_{\sigma}\right)= \\
& e_{\sigma}^{\mathrm{T}}\left(\tau_{i}^{+}\right) \Delta_{\theta_{\sigma}}^{\mathrm{T}} \bar{S} \Delta_{\theta_{\sigma}} e_{\sigma}\left(\tau_{i}^{+}\right) .
\end{aligned}
$$

We take

$$
\begin{aligned}
& H_{1, \sigma}=\theta_{\max }\left\{\Delta_{\theta_{\sigma}}^{\mathrm{T}} \bar{S} \Delta_{\theta_{\sigma}}\right\}, H_{1}=\max _{\sigma \in \Pi}\left\{H_{1, \sigma}\right\} \\
& H_{2, \sigma}=\theta_{\min }\left\{\Delta_{\theta_{\sigma}}^{\mathrm{T}} \bar{S} \Delta_{\theta_{\sigma}}\right\}, H_{2}=\min _{\sigma \in \Pi}\left\{H_{2, \sigma}\right\} .
\end{aligned}
$$

It can easily be shown that

$$
\begin{aligned}
& V_{\sigma\left(\tau_{i}^{+}\right)} \leq \\
& H_{1} e_{\sigma}^{\mathrm{T}}\left(\tau_{i}^{+}\right) e_{\sigma}\left(\tau_{i}^{+}\right) \leq \\
& H_{1}\left(\Gamma e_{\sigma}\left(\tau_{i}^{-}\right)\right)^{\mathrm{T}} \Gamma e_{\sigma}\left(\tau_{i}^{-}\right) \\
& \Gamma=I \leq \\
& \frac{H_{1}}{H_{2}} e_{\sigma}^{\mathrm{T}}\left(\tau_{i}^{-}\right) \Delta_{\theta_{\sigma}}^{\mathrm{T}} \bar{S} \Delta_{\theta_{\sigma}} e_{\sigma}\left(\tau_{i}^{-}\right) \leq \\
& \frac{H_{1}}{H_{2}} \bar{e}_{\sigma}^{\mathrm{T}}\left(\tau_{i}^{-}\right) \bar{S} e_{\sigma}\left(\tau_{i}^{-}\right) \leq \\
& H \bar{e}_{\sigma}^{\mathrm{T}}\left(\tau_{i}^{-}\right) \bar{S} e_{\sigma}\left(\tau_{i}^{-}\right)
\end{aligned}
$$

where $H>1$.

$$
V_{\sigma\left(\tau_{i}^{+}\right)} \leq H V_{\sigma\left(\tau_{i}^{-}\right)}
$$

Alternatively,

$$
V_{\sigma\left(\tau_{i+1}^{+}\right)} \leq H V_{\sigma\left(\tau_{i+1}^{-}\right)} \leq H \mathrm{e}^{-\mu\left(\tau_{i+1}-\tau_{i}\right)} V_{\sigma\left(\tau_{i}^{+}\right)} .
$$

Iterating from 0 to $M$, we find

$$
V_{\sigma} \leq H^{M} \mathrm{e}^{-\mu\left(t-t_{0}\right)} V_{\sigma\left(\tau_{0}^{+}\right)} .
$$

Finally, for $M \rightarrow \infty, V_{\sigma} \rightarrow 0$, which includes the global convergence of the error estimation and the stability of the proposed observer.

\subsection{Observer design with arbitrary switch- ing strategy}

In this part of work, we extend the previous methodology to estimate the unknown states and the discrete dynamics as explained in Fig. 1. In fact, we have to design a continuous hybrid high gain observer and a decision function which allow the estimation of the suitable mode location which ensures a better performance of the estimation error.

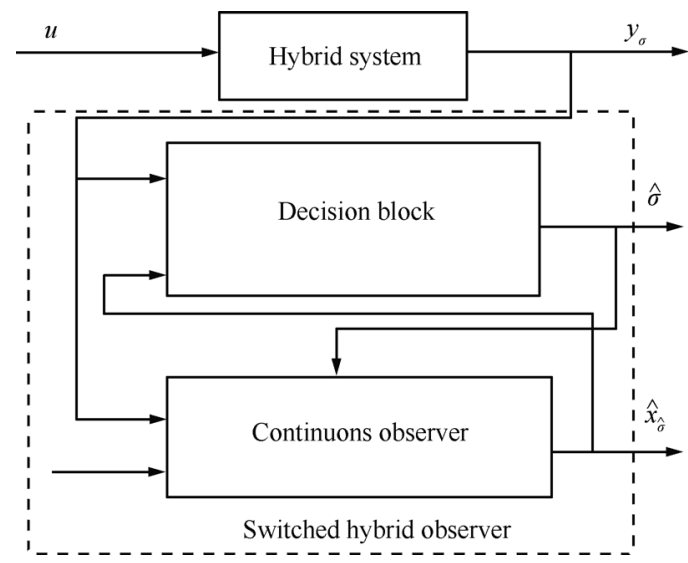

Fig. 1 The scheme of hybrid observer with recoverable switching strategy

The role of the decision function is to detect any fault during the switching from one subsystem to another. According to the information taken from a decision function, a mode location is responsible to organize the switching strategies.

The discrete estimation is done based on a multiple Lyapunov function $V_{\hat{\sigma}}$ which must decrease with time for an active subsystem. The Lyapunov function equation is obtained as

$$
V_{\hat{\sigma}}=\bar{e}^{\mathrm{T}} S \bar{e}=\left(\Delta_{\theta}\left(\hat{x}_{\hat{\sigma}}-x_{\sigma}\right)\right)^{\mathrm{T}} S\left(\Delta_{\theta}\left(\hat{x}_{\hat{\sigma}}-x_{\sigma}\right)\right) .
$$

The continuous observer for the nonlinear switched hybrid system class (1) is given by

$\sum_{\hat{\sigma}}\left\{\begin{array}{l}\dot{\hat{x}}_{\hat{\sigma}}=A \hat{x}_{\hat{\sigma}}+\varphi_{\hat{\sigma}}\left(\hat{x}_{\hat{\sigma}}, u\right)-\theta_{\hat{\sigma}} \Delta_{\theta_{\hat{\sigma}}}^{-1} S^{-1} C^{\mathrm{T}}\left(C \hat{x}_{\hat{\sigma}}-y\right) \\ \hat{\sigma}=\sigma, \quad \text { if } \quad r_{\sigma \hat{\sigma}}=\left|y_{\sigma}-C \hat{x}_{\hat{\sigma}}\right| \leq \mathrm{K}\end{array}\right.$

where $\hat{\sigma}(t) \in \prod$, the states $\hat{x}(t) \in \mathbf{R}^{n}, \hat{x}^{k} \in \mathbf{R}^{n_{k}}$, $\sum_{k=1}^{p} n_{k}=n, k=1, \cdots, q$ and $\theta_{\hat{\sigma}} \geq 0$ is a real number. 
$\Delta_{\theta_{\hat{\sigma}}}$ is the block diagonal matrix defined by

$$
\Delta_{\theta_{\hat{\sigma}}}=\operatorname{diag}\left[\begin{array}{llll}
\frac{I_{p}}{\theta_{\hat{\sigma}}} & \frac{I_{p}}{\theta_{\hat{\sigma}}^{2}} & \cdots & \frac{I_{p}}{\theta_{\hat{\sigma}}^{n}}
\end{array}\right] .
$$

Indeed, we can establish the main fundamental result.

Theorem 2. If the nonlinear switched hybrid system defined by (1) satisfies the Assumptions 1-3, then, the estimation error is globally exponentially stable for $\sigma=\hat{\sigma}$ and asymptotically stable for $\sigma \neq \hat{\sigma}$.

Proof. It is trivial that for $t=0$, the system $\left(\sum_{\hat{\sigma}}\right)$ converges exponentially to $\left(\sum_{\sigma}\right)$, and we have just to select the observer $\left(\sum_{\hat{\sigma}_{0}}\right)$. We assume that at time $t=\tau_{1}$, the system $\left(\sum_{\sigma}\right)$ jumps from $\sigma_{0}$ to mode $\sigma$. In this case, we set $e_{\sigma \hat{\sigma}}(t)=\hat{x}_{\hat{\sigma}}(t)-x_{\sigma}(t)$ to be the vector of the error between the real state vector and its estimation. We have

$$
\begin{gathered}
\dot{e}_{\sigma \hat{\sigma}}=A e_{\sigma \hat{\sigma}}+\Lambda_{\sigma \hat{\sigma}}\left(\hat{x}_{\hat{\sigma}}, x_{\sigma}\right)-\theta_{\hat{\sigma}} \Delta_{\theta_{\hat{\sigma}}}^{-1} S^{-1} C^{\mathrm{T}}\left(C \hat{x}_{\hat{\sigma}}-y\right)= \\
\left(A-\theta_{\hat{\sigma}} \Delta_{\theta_{\hat{\sigma}}}^{-1} S^{-1} C^{\mathrm{T}} C\right) e_{\sigma \hat{\sigma}}+\Lambda_{\sigma \hat{\sigma}}\left(\hat{x}_{\hat{\sigma}}, x_{\sigma}\right)
\end{gathered}
$$

with

$$
\begin{aligned}
& \Lambda_{\sigma \hat{\sigma}}\left(\hat{x}_{\hat{\sigma}}, x_{\sigma}\right)=\varphi_{\hat{\sigma}}\left(\hat{x}_{\hat{\sigma}}\right)-\varphi_{\sigma}\left(x_{\sigma}\right)= \\
& \quad\left(\varphi_{\hat{\sigma}}\left(\hat{x}_{\hat{\sigma}}\right)-\varphi_{\hat{\sigma}}\left(x_{\sigma}\right)\right)+\left(\varphi_{\hat{\sigma}}\left(x_{\sigma}\right)-\varphi_{\sigma}\left(x_{\sigma}\right)\right)= \\
& \quad \tilde{\varphi}_{x \hat{x}}+\tilde{\varphi}_{\sigma \hat{\sigma}} .
\end{aligned}
$$

We can easily verify the following identities: $\Delta_{\theta_{\hat{\sigma}}} A \Delta_{\theta_{\hat{\sigma}}}^{-1}=$ $\theta_{\hat{\sigma}} A$ and $C \Delta_{\theta_{\hat{\sigma}}}=C$.

Considering the following change of variable $\bar{e}_{\sigma \hat{\sigma}}=$ $\Delta_{\theta_{\hat{\sigma}}} e_{\sigma \hat{\sigma}}$, we get

$$
\begin{gathered}
\dot{\bar{e}}_{\sigma \hat{\sigma}}=\left(\Delta_{\theta_{\hat{\sigma}}} A \Delta_{\theta_{\hat{\sigma}}}^{-1}-\theta_{\hat{\sigma}} S^{-1} C^{\mathrm{T}} C \Delta_{\theta_{\hat{\sigma}}}^{-1}\right) \bar{e}_{\sigma \hat{\sigma}}+\Delta_{\theta_{\hat{\sigma}}} \Lambda_{\sigma \hat{\sigma}}\left(\hat{x}_{\hat{\sigma}}, x_{\sigma}\right)= \\
\theta_{\hat{\sigma}}\left(A-S^{-1} C^{\mathrm{T}} C\right) \bar{e}_{\sigma \hat{\sigma}}+\Delta_{\theta_{\hat{\sigma}}} \Lambda_{\sigma \hat{\sigma}}\left(\hat{x}_{\hat{\sigma}}, x_{\sigma}\right) .
\end{gathered}
$$

There is a symmetric positive definite matrix $S$ defined by

$$
\left(A-S^{-1} C^{\mathrm{T}} C\right)^{\mathrm{T}} S+S\left(A-S^{-1} C^{\mathrm{T}} C\right)=-I_{n} .
$$

$V_{\sigma}$ is the Lyapunov function candidate with $V_{\sigma}\left(\bar{e}_{\sigma \hat{\sigma}}\right)=$ $\bar{e}_{\sigma \hat{\sigma}}^{\mathrm{T}} S \bar{e}_{\sigma \hat{\sigma}}$.

$$
\begin{aligned}
& \dot{V}_{\sigma}\left(\bar{e}_{\sigma \hat{\sigma}}\right)=2 \bar{e}_{\sigma \hat{\sigma}}^{\mathrm{T}} S \dot{\bar{e}}_{\sigma \hat{\sigma}}= \\
& -\theta_{\hat{\sigma}} \bar{e}_{\sigma \hat{\sigma}}^{\mathrm{T}} \bar{e}_{\sigma \hat{\sigma}}+2 \bar{e}_{\sigma \hat{\sigma}}^{\mathrm{T}} S \Delta_{\theta_{\hat{\sigma}}}\left(\tilde{\varphi}_{x \hat{x}}+\tilde{\varphi}_{\sigma \hat{\sigma}}\right) .
\end{aligned}
$$

$\varphi_{\sigma}$ is Lipschitzian, then we can show that $\left\|\Delta_{\theta_{\hat{\sigma}}} \tilde{\varphi}_{x \hat{x}}\right\| \leq$ $\delta_{\sigma}\left\|\bar{e}_{\sigma \hat{\sigma}}\right\|$, where $\delta_{\sigma} \geq 0$.

$$
\left\|\bar{e}_{\sigma \hat{\sigma}}^{\mathrm{T}} S \Delta_{\theta_{\hat{\sigma}}} \tilde{\varphi}_{x \hat{x}}\right\| \leq \lambda_{\max }(S) \delta_{\sigma}\left\|\bar{e}_{\sigma \hat{\sigma}}\right\|^{2} .
$$

$\lambda_{\max }(S)$ is the largest eigenvalue of $S$. Then we can get

$$
\begin{array}{r}
\dot{V}_{\sigma}\left(\bar{e}_{\sigma \hat{\sigma}}\right) \leq-\left(\theta_{\hat{\sigma}}-2 \delta_{\sigma} \lambda_{\max }(S)\right)\left\|\bar{e}_{\sigma \hat{\sigma}}\right\|^{2}+2 \bar{e}_{\sigma \hat{\sigma}}^{\mathrm{T}} S \Delta_{\theta_{\hat{\sigma}}} \tilde{\varphi}_{\sigma \hat{\sigma}} \leq \\
\quad-\mu_{\hat{\sigma}} V_{\sigma}\left(\bar{e}_{\sigma \hat{\sigma}}\right)+\frac{2 \beta}{\theta_{\hat{\sigma}}} \sqrt{\lambda_{\max }(S)} \sqrt{V_{\sigma}\left(\bar{e}_{\sigma \hat{\sigma}}\right)}\left\|\tilde{\varphi}_{\sigma \hat{\sigma}}\right\| \quad(24)
\end{array}
$$

where $\beta_{1}$ is a positive constant and $\mu_{\hat{\sigma}}=\frac{\left(\theta_{\hat{\sigma}}-2 \delta_{\sigma} \lambda_{\max }(S)\right)}{\lambda_{\min }(S)}>$ 0, i.e., $\theta_{\hat{\sigma}}>2 \delta_{\sigma} \lambda_{\max }(S)$.

Considering $\dot{V}_{\sigma}\left(\bar{e}_{\sigma \hat{\sigma}}\right)=2 \sqrt{V_{\sigma}\left(\bar{e}_{\sigma \hat{\sigma}}\right)} \sqrt{V_{\sigma}\left(\bar{e}_{\sigma \hat{\sigma}}\right)}$, we find

$$
\sqrt{V_{\sigma}\left(\bar{e}_{\sigma \hat{\sigma}}\right)} \leq-\mu_{\hat{\sigma}} \sqrt{V_{\sigma}\left(\bar{e}_{\sigma \hat{\sigma}}\right)}+\frac{2 \beta_{1}}{\theta_{\hat{\sigma}}} \sqrt{\lambda_{\max }(S)}\left\|\tilde{\varphi}_{\sigma \hat{\sigma}}\right\|
$$

If $\hat{\sigma}=\sigma$, then $\tilde{\varphi}_{\sigma \hat{\sigma}}=0$,

$$
\sqrt{V_{\sigma}\left(\bar{e}_{\sigma \sigma}(t)\right)} \leq \mathrm{e}^{-\mu_{\sigma}\left(t-t_{0}\right)} \sqrt{V_{\sigma}\left(\bar{e}_{\sigma \sigma}\left(t_{0}\right)\right)} .
$$

Supposing that $\lambda=\frac{\sqrt{\lambda_{\max }(S)}}{\sqrt{\lambda_{\min }(S)}}$, we have

$$
\begin{aligned}
& \left\|\bar{e}_{\sigma \sigma}(t)\right\| \leq \frac{\sqrt{\lambda_{\max }(S)}}{\sqrt{\lambda_{\min }(S)}} \mathrm{e}^{-\mu_{\sigma}\left(t-t_{0}\right)}\left\|\bar{e}_{\sigma \sigma}\left(t_{0}\right)\right\| \\
& \left\|\Delta_{\theta_{\sigma}} e_{\sigma \sigma}(t)\right\| \leq \lambda \mathrm{e}^{-\mu_{\sigma}\left(t-t_{0}\right)}\left\|e_{\sigma \sigma}\left(t_{0}\right)\right\| .
\end{aligned}
$$

For the residual $r_{\sigma \sigma}$, we can get

$$
r_{\sigma \sigma}(t)=\left|C e_{\sigma \sigma}\right|=\left|e_{1, \sigma \sigma}\right| \leq\left\|\Delta_{\theta_{\sigma}} e_{\sigma \sigma}(t)\right\| .
$$

It is easily shown that there exists a time $t=\tau$ verifying

$$
r_{\sigma \sigma}(\tau)=\lambda \mathrm{e}^{-\mu_{\sigma}\left(\tau-t_{0}\right)}\left\|e_{\sigma \sigma}\left(t_{0}\right)\right\| \leq K .
$$

On the other hand, if $\hat{\sigma} \neq \sigma$, we have

$$
\sqrt{V_{\sigma}\left(\bar{e}_{\sigma \hat{\sigma}}(t)\right)} \leq \mu_{\hat{\sigma}} \sqrt{V_{\sigma}\left(\bar{e}_{\sigma \sigma}\left(t_{0}\right)\right.}+\frac{\beta_{2}}{\theta_{\hat{\sigma}}}
$$

where $\beta_{2}=2 \rho \beta_{1} \sqrt{\lambda_{\max }(S)}$ and $\rho$ is the upper bound of $\tilde{\varphi}_{\sigma \hat{\sigma}}$.

$$
\begin{aligned}
& \sqrt{V_{\sigma}\left(\bar{e}_{\sigma \hat{\sigma}}(t)\right)} \leq \\
& \mathrm{e}^{-\mu_{\hat{\sigma}}\left(t-t_{0}\right)} \sqrt{V_{\sigma}\left(\bar{e}_{\sigma \hat{\sigma}}\left(t_{0}\right)\right.}+\frac{\beta_{2}}{\theta_{\hat{\sigma}}} \mathrm{e}^{-\mu_{\hat{\sigma}} t} \int_{t_{0}}^{t} \mathrm{e}^{-\mu_{\hat{\sigma}} s} \mathrm{~d} s \leq \\
& \mathrm{e}^{-\mu_{\hat{\sigma}}\left(t-t_{0}\right)} \sqrt{V_{\sigma}\left(\bar{e}_{\sigma \hat{\sigma}}\left(t_{0}\right)\right)}+\frac{\beta_{2}}{\theta_{\hat{\sigma}} \mu_{\hat{\sigma}}}\left[1-\mathrm{e}^{-\mu_{\hat{\sigma}}\left(t-t_{0}\right)}\right] .
\end{aligned}
$$

In the same manner as before, we get

$$
\begin{gathered}
\left\|\bar{e}_{\sigma \hat{\sigma}}(t)\right\| \leq \frac{\sqrt{\lambda_{\max }(S)}}{\sqrt{\lambda_{\min }(S)}} \mathrm{e}^{-\mu_{\hat{\sigma}}\left(t-t_{0}\right)}\left\|\bar{e}_{\sigma \hat{\sigma}}\left(t_{0}\right)\right\|+ \\
\frac{\beta_{2}}{\theta_{\hat{\sigma}} \mu_{\hat{\sigma}}}\left[1-\mathrm{e}^{-\mu_{\hat{\sigma}}\left(t-t_{0}\right)}\right] \\
\left\|\Delta_{\theta_{\hat{\sigma}}} e_{\sigma \hat{\sigma}}(t)\right\| \leq \lambda \mathrm{e}^{-\mu_{\hat{\sigma}}\left(t-t_{0}\right)}\left\|e_{\sigma \hat{\sigma}}\left(t_{0}\right)\right\|+ \\
\frac{\beta_{2}}{\theta_{\hat{\sigma}} \mu_{\hat{\sigma}}}\left[1-\mathrm{e}^{-\mu_{\hat{\sigma}}\left(t-t_{0}\right)}\right] .
\end{gathered}
$$

Since $\theta_{\hat{\sigma}}>1$, we get

$$
\begin{aligned}
& r_{\sigma \hat{\sigma}}(t)=\left|e_{1, \sigma \hat{\sigma}}\right| \leq\left\|\Delta_{\theta_{\hat{\sigma}}} e_{\sigma \hat{\sigma}}(t)\right\| \leq \\
& \quad \lambda \mathrm{e}^{-\mu_{\hat{\sigma}}\left(t-t_{0}\right)}\left\|e_{\sigma \hat{\sigma}}\left(t_{0}\right)\right\|+\frac{\beta_{2}}{\mu_{\hat{\sigma}}}\left[1-\mathrm{e}^{-\mu_{\hat{\sigma}}\left(t-t_{0}\right)}\right] .
\end{aligned}
$$

We want to ensure that if $t \geq \tau$, we have $r_{\sigma \hat{\sigma}}(t) \geq K$. When $t \rightarrow+\infty$, we can verify that $r_{\sigma \hat{\sigma}}(t) \leq \frac{\beta_{2}}{\mu_{\hat{\sigma}}}$. We must choose $K$ as

$$
K<\frac{\beta_{2}}{\mu_{\hat{\sigma}}}
$$

As before, it is assumed that $e_{\hat{\sigma} \hat{\sigma}}\left(\tau_{i}^{+}\right)=\Gamma e_{\sigma \sigma}\left(\tau_{i}^{-}\right)$supposing that $\Gamma=I$, then we have no jumps.

For $t \in\left[\tau_{i-1}, \tau_{i}\right]$, we have

$$
V_{\sigma}\left(\bar{e}_{\sigma \sigma}\left(\tau_{i}^{-}\right)\right) \leq \mathrm{e}^{-\mu\left(\tau_{i}-\tau_{i-1}\right)} V_{\sigma}\left(\bar{e}_{\sigma \sigma}\left(\tau_{i-1}^{+}\right)\right) .
$$

For $\left[\tau_{i}, \tau_{i+1}\right]$

$$
V_{\hat{\sigma}}\left(\bar{e}_{\hat{\sigma} \hat{\sigma}}\left(\tau_{i+1}^{-}\right)\right) \leq \mathrm{e}^{-\mu\left(\tau_{i+1}-\tau_{i}\right)} V_{\hat{\sigma}}\left(\bar{e}_{\hat{\sigma} \hat{\sigma}}\left(\tau_{i}^{+}\right)\right) .
$$


Supposing that $e_{\hat{\sigma} \hat{\sigma}}\left(\tau_{i}^{+}\right)=\Gamma e_{\sigma \sigma}\left(\tau_{i}^{-}\right)$, then $\bar{e}_{\hat{\sigma} \hat{\sigma}}=\Delta_{\theta_{\hat{\sigma}}} e_{\hat{\sigma} \hat{\sigma}}$

$$
\begin{gathered}
V_{\hat{\sigma}}\left(\bar{e}_{\hat{\sigma} \hat{\sigma}}\left(\tau_{i+1}^{-}\right)\right) \leq \mathrm{e}^{-\mu\left(\tau_{i+1}-\tau_{i}\right)} V_{\hat{\sigma}}\left(\bar{e}_{\hat{\sigma} \hat{\sigma}}\left(\tau_{i}^{+}\right)\right) \leq \\
\mathrm{e}^{-\mu\left(\tau_{i+1}-\tau_{i}\right)} \Delta_{\theta_{\hat{\sigma}}} \mathrm{e}_{\hat{\sigma} \hat{\sigma}}\left(\tau_{i}^{+}\right)^{\mathrm{T}} S \Delta_{\theta_{\hat{\sigma}}} e_{\hat{\sigma} \hat{\sigma}}\left(\tau_{i}^{+}\right) \leq \\
\mathrm{e}^{-\mu\left(\tau_{i+1}-\tau_{i}\right)} \lambda_{\max }(S)\left\|\mathrm{e}_{\hat{\sigma} \hat{\sigma}}\left(\tau_{i}^{+}\right)\right\|^{2} .
\end{gathered}
$$

Since $\theta_{\hat{\sigma}}>1$ and $\Gamma=I$, we get

$$
\begin{gathered}
V_{\hat{\sigma}}\left(\bar{e}_{\hat{\sigma} \hat{\sigma}}\left(\tau_{i+1}^{-}\right)\right) \leq \mathrm{e}^{-\mu\left(\tau_{i+1}-\tau_{i}\right)} \frac{\lambda_{\max }(S)}{\theta_{\hat{\sigma}}}\left\|e_{\hat{\sigma} \hat{\sigma}}\left(\tau_{i}^{+}\right)\right\|^{2} \leq \\
\mathrm{e}^{-\mu\left(\tau_{i+1}-\tau_{i}\right)} \frac{\lambda_{\max }(S)}{\theta_{\hat{\sigma}}}\left\|\Gamma e_{\sigma \sigma}\left(\tau_{i}^{-}\right)\right\|^{2} .
\end{gathered}
$$

Alternatively,

$$
\begin{gathered}
V_{\hat{\sigma}}\left(\bar{e}_{\hat{\sigma} \hat{\sigma}}\left(\tau_{i+1}^{-}\right)\right) \leq \mathrm{e}^{-\mu\left(\tau_{i+1}-\tau_{i}\right)} \frac{\theta_{\hat{\sigma}}^{2 n} \lambda_{\max }(S)}{\theta_{\hat{\sigma}}}\left\|\Delta_{\theta_{\sigma}} e_{\sigma \sigma}\left(\tau_{i}^{-}\right)\right\| \leq \\
\mathrm{e}^{-\mu\left(\tau_{i+1}-\tau_{i}\right)} \frac{\theta_{\hat{\sigma}}^{2 n} \lambda_{\max }(S)}{\theta_{\hat{\sigma}} \lambda_{\min }(S)} V_{\sigma}\left(\bar{e}_{\sigma \sigma}\left(\tau_{i}^{-}\right)\right) \leq \\
H \mathrm{e}^{-\mu\left(\tau_{i+1}-\tau_{i}\right)} V_{\sigma}\left(\bar{e}_{\sigma \sigma}\left(\tau_{i}^{-}\right)\right)
\end{gathered}
$$

with $H>1$, we have

$$
V_{\hat{\sigma}}\left(\bar{e}_{\hat{\sigma} \hat{\sigma}}\left(\tau_{i+1}^{-}\right)\right) \leq H \mathrm{e}^{-\mu\left(\tau_{i+1}-\tau_{i}\right)} \mathrm{e}^{-\mu\left(\tau_{i}-\tau_{i-1}\right)} V_{\sigma}\left(e_{\sigma \sigma}\left(\tau_{i-1}^{+}\right)\right) .
$$

Iterating from $i=1, \cdots, M$, we conclude

$$
V_{\hat{\sigma}}\left(\bar{e}_{\hat{\sigma} \hat{\sigma}}\left(\tau_{i+1}^{-}\right)\right) \leq H^{M} \mathrm{e}^{-\mu\left(\tau_{i+1}-\tau_{0}\right)} V_{\sigma_{0}}\left(e_{\sigma_{0} \sigma_{0}}\left(\tau_{0}^{+}\right)\right) .
$$

\section{Simulation example}

\subsection{Description of a quadruple tank pro- cess}

In this section, we present a real process which can be described as a nonlinear switched system (1). The system is a multivariable process which consists of four interconnected water tanks as shown in Fig. $2^{[22]}$. This system is considered as a suitable model that shows the applicability of our results.

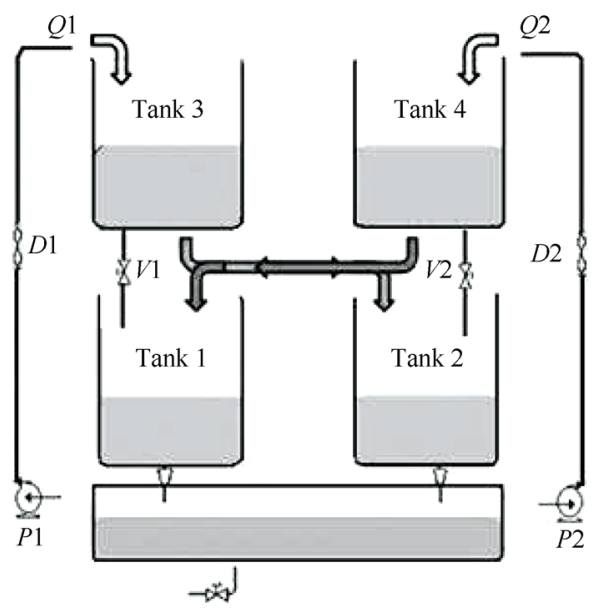

Fig. 2 The quadruple tank process
The flows of the independent pumps $\left(P_{1}, P_{2}\right)$ are the two inputs $\left(Q_{1}, Q_{2}\right)$ of the quadruple tank process and the levels in the four tanks $\left(h_{1}, h_{2}, h_{3}, h_{4}\right)$ are the outputs. The two pumps are used to transfer liquid from a basin into tanks 3 and 4, respectively. They drain freely into tanks 1 and 2 , respectively. Only the liquid levels in the bottom two tanks are measured using ultrasonic sensors which involve the necessity of the observers to estimate the unknown liquid levels in the upper tanks 3 and 4 .

The differential equations that describe the hybrid system dynamics are given by

$$
\begin{gathered}
\sum_{1}\left(\begin{array}{l}
\dot{x}_{1}=-C_{1} \sqrt{x_{1}}+C_{2} \sqrt{x_{3}}+C_{3} \sqrt{x_{4}} \\
\dot{x}_{2}=-C_{4} \sqrt{x_{2}}+C_{5} \sqrt{x_{3}}+C_{6} \sqrt{x_{4}} \\
\dot{x}_{3}=-C_{7} \sqrt{x_{3}}+C_{8} u_{1} \\
\dot{x}_{4}=-C_{9} \sqrt{x_{4}}+C_{10} u_{2}
\end{array}\right) \\
\sum_{2}\left(\begin{array}{l}
\dot{x}_{1}=-C_{1} \sqrt{x_{1}}+C_{2} \sqrt{x_{3}} \\
\dot{x}_{2}=-C_{4} \sqrt{x_{2}}+C_{6} \sqrt{x_{4}} \\
\dot{x}_{3}=-C_{7} \sqrt{x_{3}}+C_{8} u_{1} \\
\dot{x}_{4}=-C_{9} \sqrt{x_{4}}+C_{10} u_{2}
\end{array}\right) \\
\sum_{3}\left(\begin{array}{l}
\dot{x}_{1}=-C_{1} \sqrt{x_{1}}+C_{3} \sqrt{x_{4}} \\
\dot{x}_{2}=-C_{4} \sqrt{x_{2}}+C_{5} \sqrt{x_{3}} \\
\dot{x}_{3}=-C_{7} \sqrt{x_{3}}+C_{8} u_{1} \\
\dot{x}_{4}=-C_{9} \sqrt{x_{4}}+C_{10} u_{2}
\end{array}\right)
\end{gathered}
$$

where $y(t)=\left(x_{1}(t) \quad x_{2}(t)\right)^{\mathrm{T}} \in \mathbf{R}^{2}$ is the output vector for all subsystems, the state vector $x(t) \in \mathbf{R}^{4}$ represents the liquid levels in different tanks for every subsystem, $u(t)=$ $\left(u_{1}(t) \quad u_{2}(t)\right)^{\mathrm{T}} \in \mathbf{R}^{2}$ denotes the flow of every pump and the constants of the system are $C_{i}, i=1, \cdots, 10$.

To put the models of the quadruple tank process (35)(37) under the nonlinear system class (1), one shall apply the following changes of coordinates for the models with $p=2$ and $q=2$.

$$
\begin{aligned}
& \text { For model 1, }\left(\begin{array}{l}
X_{1}=x_{1} \\
X_{2}=x_{2} \\
X_{3}=C_{2} \sqrt{x_{3}}+C_{3} \sqrt{x_{4}} \\
X_{4}=C_{5} \sqrt{x_{3}}+C_{6} \sqrt{x_{4}}
\end{array}\right) \\
& \text { For model 2, }\left(\begin{array}{l}
X_{1}=x_{1} \\
X_{2}=x_{2} \\
X_{4}=C_{2} \sqrt{x_{3}} \\
X_{4}=C_{6} \sqrt{x_{4}}
\end{array}\right) \\
& \text { For model 3, }\left(\begin{array}{l}
X_{1}=x_{1} \\
X_{2}=x_{2} \\
X_{3}=C_{3} \sqrt{x_{4}} \\
X_{4}=C_{5} \sqrt{x_{3}}
\end{array}\right) .
\end{aligned}
$$

We can deduce the models of the quadruple tank process in the new representations with $\dot{X}_{j}=\frac{\partial X_{j}}{\partial t}, j=1, \cdots, 4$. 


$$
\sum_{1}\left\{\begin{aligned}
\dot{X}_{1}= & X_{3}-C_{1} \sqrt{X_{1}} \\
\dot{X}_{2}= & X_{4}-C_{4} \sqrt{X_{2}} \\
\dot{X}_{3}= & -\frac{C_{2} C_{7}+C_{3} C_{9}}{2}+\frac{C_{2} C_{6}-C_{3} C_{5}}{2} \times \\
& {\left[\frac{C_{2} C_{8}}{C_{6} X_{3}-C_{3} X_{4}} u_{1}-\frac{C_{3} C_{10}}{C_{5} X_{3}-C_{2} X_{4}} u_{2}\right] } \\
\dot{X}_{4}= & -\frac{C_{5} C_{7}+C_{6} C_{9}}{2}+\frac{C_{2} C_{6}-C_{3} C_{5}}{2} \times \\
& {\left[\frac{C_{5} C_{8}}{C_{6} X_{3}-C_{3} X_{4}} u_{1}-\frac{C_{6} C_{10}}{C_{5} X_{3}-C_{2} X_{4}} u_{2}\right] }
\end{aligned}\right.
$$

$\sum_{2}\left\{\begin{array}{l}\dot{X}_{1}=X_{3}-C_{1} \sqrt{X_{1}} \\ \dot{X}_{2}=X_{4}-C_{4} \sqrt{X_{2}} \\ \dot{X}_{3}=-\frac{C_{2}^{2}}{2}+\frac{C_{2}^{2} C_{8} u_{1}}{2 X_{3}} \\ \dot{X}_{4}=-\frac{C_{6}^{2}}{2}+\frac{C_{6}^{2} C_{10} u_{2}}{2 X_{4}}\end{array}\right.$

$$
\sum_{3}\left\{\begin{array}{l}
\dot{X}_{1}=X_{3}-C_{1} \sqrt{X_{1}} \\
\dot{X}_{2}=X_{4}-C_{4} \sqrt{X_{2}} \\
\dot{X}_{3}=-\frac{C_{3}^{2}}{2}+\frac{C_{3}^{2} C_{10} u_{2}}{2 X_{3}} \\
\dot{X}_{4}=-\frac{C_{5}^{2}}{2}+\frac{C_{5}^{2} C_{8} u_{1}}{2 X_{4}}
\end{array}\right.
$$

The system output vector is $y(t)=\left(\begin{array}{ll}X_{1}(t) & X_{2}(t)\end{array}\right)^{\mathrm{T}}$.

\subsection{Simulation results}

We apply the discrete-continuous observers previously described to estimate the unknown states $\left(X_{3}, X_{4}\right)$. For comparison purposes, we consider the function of the switching strategy $\sigma(t)$ to be fixed, and then it must be estimated. The initial conditions of the real and estimated states are $X(0)=\left[10^{-3} ; 10^{-3} ; 10 ; 12\right]$ and $\hat{X}(0)=$ $\left[10^{-3} ; 10^{-3} ; 5 ; 4\right]$, respectively. The inputs are bounded by $u_{\max }=15 \mathrm{ml} / \mathrm{s}$. We have to choose the optimal values of the design parameters for different mode locations $\{1,2,3\}$.

To check the robustness of the presented observers, all subsystem outputs are assumed corrupted with uniform white Gaussian noise signal of magnitude $4 \times 10^{-3}$. The actual mode location $\sigma(t)$ and the discrete estimation $\hat{\sigma}(t)$ are drawn in Fig. 3 which show a satisfactory estimation of the commutation strategy. The evolution of the actual and estimated states $\left(X_{3}, X_{4}\right)$ and their errors are depicted in Figs. 4 and 5, respectively. In fact, the error dynamics given in Figs. 4 (b) and 5 (b) show the convergence of $e_{i, \sigma}$ and $e_{i, \hat{\sigma}}, i=3,4$, towards zero in finite time.

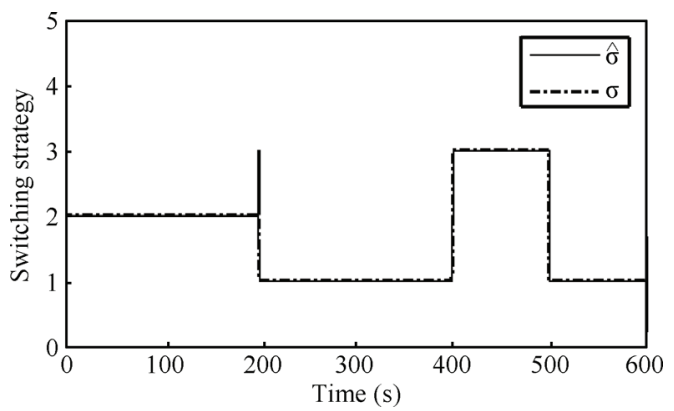

Fig. 3 The actual mode location $\sigma$ and its estimate $\hat{\sigma}$

The performance of the observers with respect to the white Gaussian noise is fairly good despite that such high gain type of observer will generally amplify the noise. Besides, the choice of $\theta_{\hat{\sigma}}$ ensures the fast convergence and the good speed of the observers. The simulation of the hybrid switched observer proves the effectiveness of such observer to estimate the continuous state of the system so as to give a satisfactory convergence of the transient value estimation.

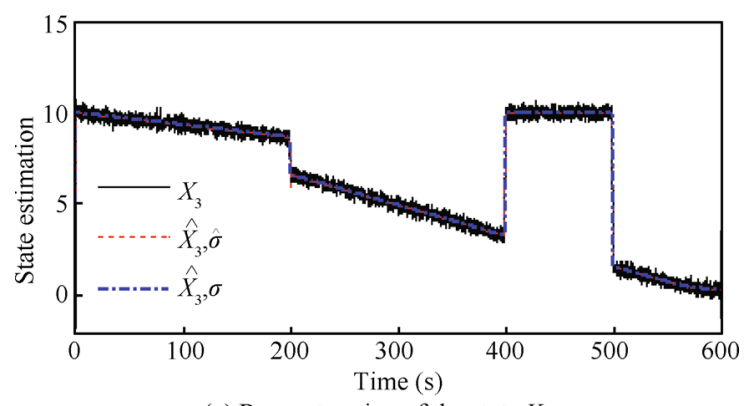

(a) Reconstruction of the state $X_{3}$

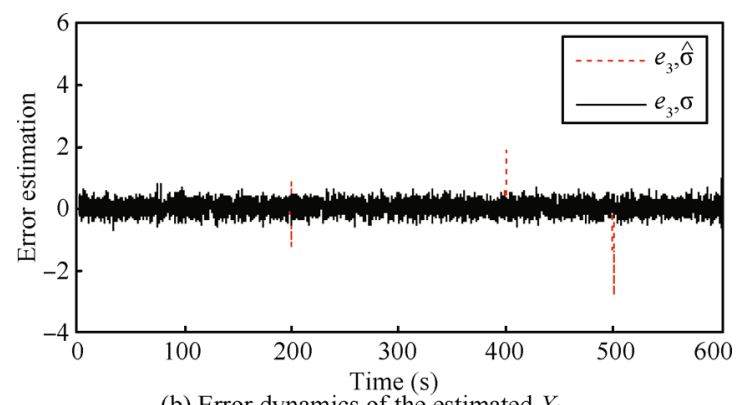

(b) Error dynamics of the estimated $X_{3}$

Fig. 4 Reconstruction of the state $X_{3}$ with relative error and its error observation

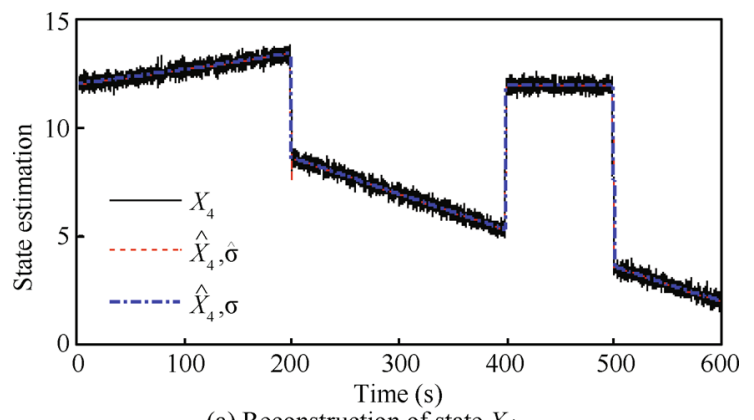

(a) Reconstruction of state $X_{4}$ 


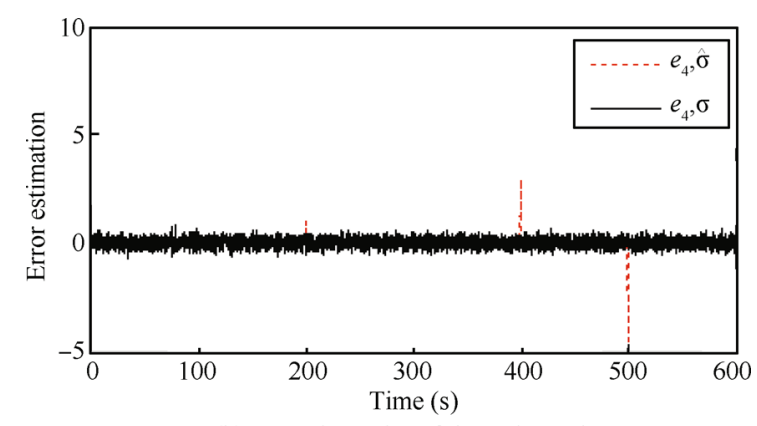

(b) Error dynamics of the estimated state $X_{4}$

Fig. 5 Reconstruction of the state $X_{4}$ with relative error and its error observation

Remark 1. Fig. 3 shows the similarity of the results of fixed and variable switching strategies. That explains the same speed of the trajectories of $\hat{X}_{i, \sigma}$ and $\hat{X}_{i, \hat{\sigma}}, i=3,4$.

\section{Conclusions}

In this paper, the problem of the estimation of hybrid states for a large class of MIMO uniformly observable nonlinear switched systems is considered. In the first case, a hybrid observer is designed considering that the switching strategy is fixed previously. Then, a discrete estimation is made taking into account the discrete dynamics of the system. Once the active mode is obtained, the continuous state is estimated. The estimation technique proposed is composed of a discrete and a continuous observer in interaction. The stability analysis is provided for the two cases using Lyapunov functions to guarantee the convergence of the estimation errors. The observers are applied to a quadruple tank process that is modeled as a nonlinear hybrid system. The simulation results show a satisfactory discrete-continuous estimation, which prove the efficiency of the designed observer in spite of the presence of noise.

\section{References}

[1] P. J. Antsaklis. A brief introduction to the theory and applications of hybrid systems. Proceedings of the IEEE vol. 88, no. 7 , pp. $879-889,2000$.

[2] S. Pettersson, B. Lennartson. Modelling, analysis and synthesis of hybrid systems. In Proceedings of Conference on Preprints of Reglernote, Lulua, Sweden, 1996.

[3] S. S. Ge., Z. D. Sun. Switched Linear Systems: Control and Design, London, UK: Springer, 2005.

[4] M. S. Branicky. Multiple Lyapunov functions and other analysis tools for switched and hybrid system. IEEE Transactions on Automatic Control, vol. 43, no. 4, pp.475-482, 1998.

[5] D. Liberzon, A. S. Morse. Basic problems in stability and design of switched systems. IEEE Control Systems, vol. 19, no. 5, pp. 59-70, 1999.
[6] X. L. Liang, M. Z. Hou, G. R. Duan. Output feedback stabilization of switched stochastic nonlinear systems under arbitrary switchings. International Journal of Automation and Computing, vol. 10, no. 6, pp. 571-577, 2013.

[7] A. Alessendri, P. Coletta. Design of Luenberger observers for a class of hybrid linear systems. Hybrid Systems: Computation and Control, Lecture Notes in Computer Science, vol. 2034, pp. 7-18, 2001.

[8] M. De La Sen, N. S. Luo. Design of linear observers for a class of linear hybrid systems. International Journal of Systems Science, vol. 31, no. 9, pp. 1077-1099, 2000.

[9] A. L. Juloski, W. P. M. H. Heemels, Y. Boers, F. Verschure. Two approaches to state estimation for a class of piecewise affine systems. In Proceedings of the 42nd Conference on Decision and Control, IEEE, Maul, USA, vol.1, pp. 143148, 2003.

[10] Z. G. Lia, C. Y. Wenb, Y. C. Sohb. Observer-based stabilization of switching linear systems. Automatica, vol. 39, no. 3, pp. 517-524, 2003.

[11] A. Balluchi, L. Benvenuti, M. D. Di Benedetto, A. L. Sangiovanni-Vincentelli. Design of observers for hybrid system. Hybrid Systems: Computation and Control, Lecture Notes in Computer Science, vol.2289, pp. 76-89, 2002.

[12] F. Hamdia, N. Manamannia, N. Messai, K. Benmahammed. Hybrid observer design for linear switched system via Differential Petri Nets. Nonlinear Analysis: Hybrid Systems, vol. 3, no. 3, pp. 310-322, 2009.

[13] A. Alessandri, P. Coletta. Switching observers for continuous-time and discrete-time linear systems. In Proceedings of the American Control Conference, IEEE, Arlington, USA, vol. 3, pp. 2516-2521, 2001.

[14] S. Pettersson. Observer design for switched systems using multiple quadratic Lyapunov functions. In Proceedings of IEEE International Symposium on Mediterrean Conference on Control and Automation Intelligent Control, IEEE, Limassol, Cyprus, pp. 262-267, 2005.

[15] M. Djemai, N. Manamani, J. P. Barbot. Sliding mode observer for triangular input hybrid system. In Proceedings of the 16th IFAC World Congress, IFAC, Prague, Czekh, vol. 16, no. 1, pp. 314-319, 2005.

[16] H. Saadaoui, N. Manamanni, M. Djemai, J. P. Barbot, T. Floquet. Exact differentiation and sliding mode observer for switched Lagrangian systems. Nonlinear Analysis: Theory, Methods and Applications, vol.65, no. 5, pp.1050-1069, 2006 .

[17] J. P. Barbot, H. Saadaoui, M. Djemai, N. Manamani. Nonlinear observer for autonomous switching systems with jumps. Nonlinear Analysis: Hybrid Systems, vol.1, no. 4, pp. 537-547, 2007.

[18] M. Farza, M. M'Saad, M. Sekher. A set of observers for a class of nonlinear systems. In Proceedings of the 16th IFAC World Congress, Praque, Czekh vol. 16, no. 1, pp. 782-782, 2005. 
[19] J. P. Gauthier, H. Hammouri, S. Othman. A simple observer for nonlinear systems-applications to bioreactors. IEEE Transactions on Control Systems Technology, vol. 37, no. 6 , pp. 875-880, 1992.

[20] H. Beikzadeh, H. D. Taghirad. Exponential nonlinear observer based on the differential state-dependent Riccati equation. International Journal of Automation and Computing, vol. 9, no. 4, pp. 358-368, 2012.

[21] N. Barhoumi, F. M'Sahli, M. Djemai, K. Busawon. Observer design for some classes of uniformly observable nonlinear hybrid systems. Nonlinear Analysis: Hybrid Systems, vol. 6, no. 4, pp. 917-929, 2012

[22] K. H. Johansson. The quadruple-tank process: A multivariable laboratory process with an adjustable zero. IEEE Transactions on Control Systems Technology, vol. 8, no. 3 , pp. 456-465, 2000.

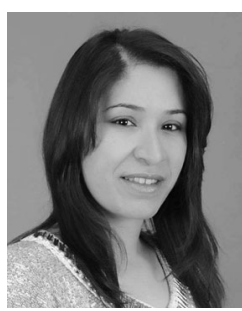

Imen Manaa graduated from the National Engineering School of Gabes (ENIG), Tunisia in 2007. She received the M. Sc. degree in electrical engineering from the National Engineering School of Monastir (ENIM), Tunisia in 2009. She is currently a $\mathrm{Ph}$. D. candidate and a member of the research unit of Study of Industrial Systems and Renewable Energy, ENIM, Tunsia. She is currently a contractual assistant professor at the Higher Institute of Computing and Techniques of Communication, Hammam-Sousse, Tunisia.

Her research interests include sliding mode control and nonlinear observers for hybrid systems.

E-mail: manaa.imen@planet.tn (Corresponding author)
ORCID iD: 0000-0002-5686-3919

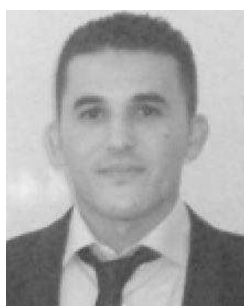

Nabil Barhoumi graduated from the National Engineering School of Gabes (ENIG), Tunisia in 2005. He received the M. Sc. degree in automatic control from the INSAT, Tunisia in 2007. He is a member of the research unit of Study of Industrial Systems and Renewable Energy. He obtained the $\mathrm{Ph} . \mathrm{D}$. degree in electrical engineering from ENIM, Tunsia. He is currently an assistant professor at the Higher Institute of Applied Sciences and Technologies of Sousse, Tunisia.

His research interests include predictive control and nonlinear observers for hybrid systems.

E-mail: nabil.barhoumi@enim.rnu.tn

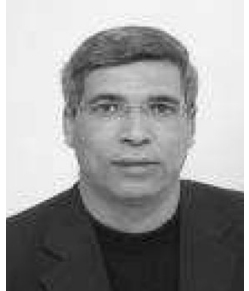

Faouzi Msahli received the M.Sc. degree of and the DEA from the High Normal School of Technical Education (ENSET), Tunisia in 1987 and 1989, respectively. He obtained the Ph. D. and the Higher Degree Research (HdR) degrees in electrical engineering from the National Engineering School of Tunis, Tunisia, in 1995 and 2001 respectively. He is the co-author of the book Identification and Digital Control of Industrial Processes, Paris, France. He is currently a professor of automatic control, ENIM, Tunisia. He is the co-responsible of the research unit of Study of Industrial Systems and Renewable Energy and the vice president of the Tunisian Association of Numerical Techniques and Automatic Control (ATTNA).

His research interests include modeling, identification, predictive and adaptive control of linear and nonlinear systems.

E-mail: faouzi.msahli@enim.rnu.tn 